Document downloaded from:

http://hdl.handle.net/10251/44148

This paper must be cited as:

Benítez López, J.; Cvetkovic-llic, D. (2013). Equalities of ideals associated with two projections in rings with involution. Linear and Multilinear Algebra. 61(10):1419-1435. doi:10.1080/03081087.2012.743026.

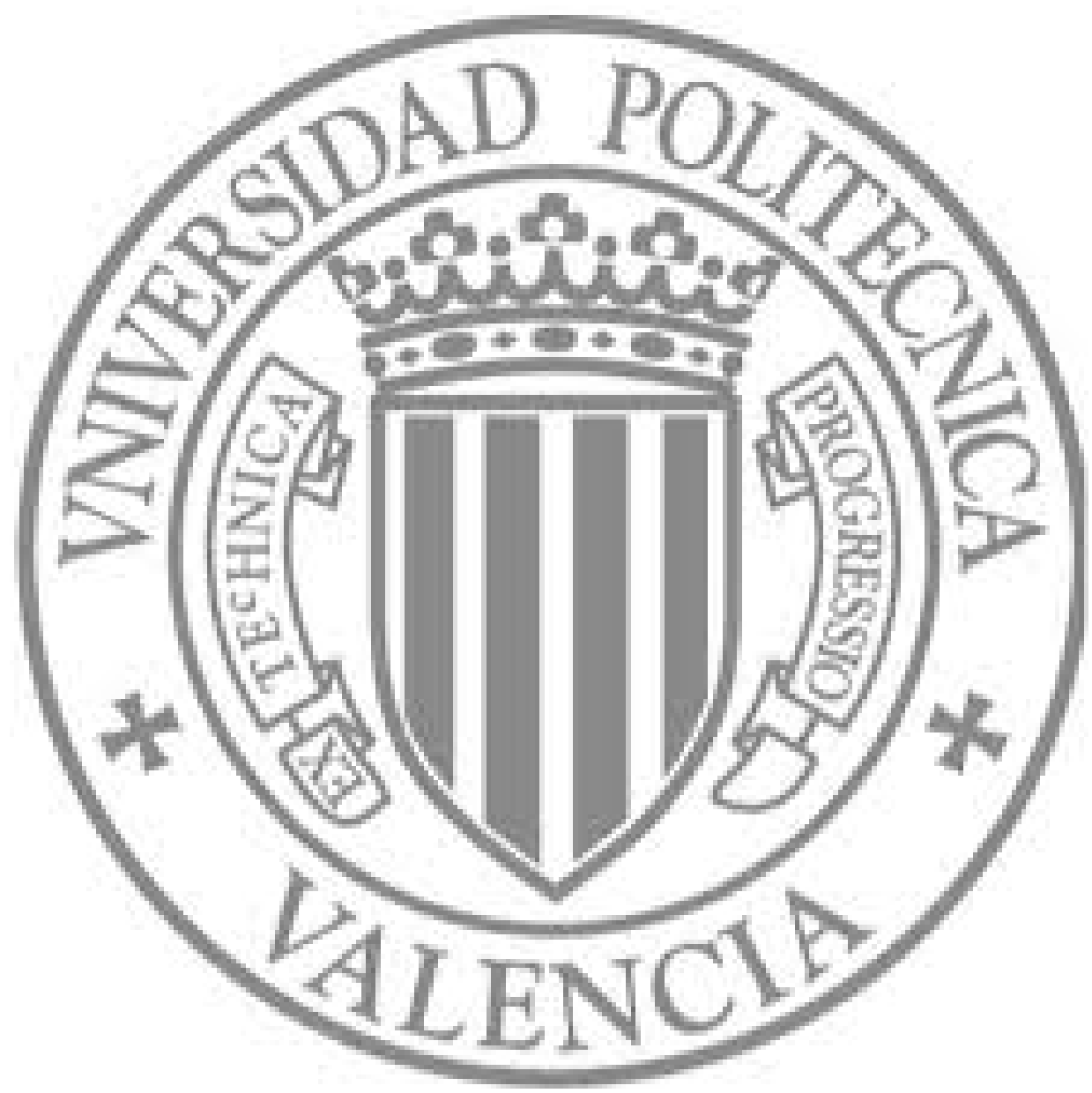

The final publication is available at

http://dx.doi.org/10.1080/03081087.2012.743026

Copyright Taylor \& Francis (Routledge): STM, Behavioural Science and Public Health Title 


\title{
Equalities of ideals associated with two projections in rings with involution
}

\author{
Julio Benítez ${ }^{*}$ and Dragana Cvetković-Ilić ${ }^{\dagger}$
}

\begin{abstract}
In this paper we study various right ideals associated with two projections (self-adjoint idempotents) in a ring with involution. Results of O.M. Baksalary, G. Trenkler, R. Piziak, P.L. Odell, and R. Hahn about orthogonal projectors (complex matrices which are Hermitian and idempotent) are considered in the setting of rings with involution. New proofs based on algebraic arguments; rather than finite-dimensional and rank theory; are given.
\end{abstract}

AMS classification: $16 \mathrm{~W} 10,16 \mathrm{D} 25$

Key words: Rings with involution; projections; Moore-Penrose inverse

\section{Introduction}

Throughout this paper, the symbol $\mathcal{R}$ will denote a unital ring ( 1 will be its unit) with an involution and the term ring will mean unital ring. Let us recall that an involution in a ring $\mathcal{R}$ is a map $a \mapsto a^{*}$ in $\mathcal{R}$ such that $(a+b)^{*}=a^{*}+b^{*},(a b)^{*}=b^{*} a^{*}$ and $\left(a^{*}\right)^{*}=a$ for any $a, b \in \mathcal{R}$. The word 'projection' will be reserved for an element $q$ of $\mathcal{R}$ which is self-adjoint and

*jbenitez@mat.upv.es. Instituto de Matemática Multidisciplinar, Universitat Politècnica de València, Camino de Vera s/n, 46022 Valencia, Spain

†dragana@pmf.ni.ac.rs. Faculty of Sciences and Mathematics, Department of Mathematics, University of Nis, 18000 Nis, Serbia, Supported by Grant No. 174007 of the Ministry of Science, Technology and Development, Republic of Serbia 
idempotent, that is $q^{*}=q=q^{2}$. With each element $a \in \mathcal{R}$ we associate an image ideal $a \mathcal{R}=\{a x: x \in \mathcal{R}\}$, and a kernel ideal $a^{\circ}=\{x \in \mathcal{R}: a x=0\}$

The paper studies some ideals and functions depending on two projections of a ring with an involution. The results given here generalize to several considered in $[1,14]$. It is worthy to note that in the proofs of those results, matrix theory is used (specifically, rank theory and singular value decomposition). We believe that giving simpler and algebraic proofs (our proofs only use algebraic reasonings) gives a greater insight of the problems considered here. We will notably consider the case of a $*$-reducing ring (where there is an implication $a^{*} a=0 \Rightarrow a=0$ for all $\left.a \in \mathcal{R}\right)$. One of the most important tools in this case is the Moore-Penrose inverse theory, which will be quickly revised together with useful results in section 2 . Section 3 then studies invertibility of $p+q$ and $p-q$ in rings with involution, together with ideal properties. Section 4 finally focuses on $*$-reducing rings. In this case, existence of particular Moore-Penrose inverses leads to certain ideal equalities.

\section{Moore-Penrose inverse and auxiliary lemmas}

The link between generalized inverses and range or kernel ideals is not new. See for instance $[4,5]$ in the case of semigroups, [13] in rings or $[6,7]$ in $\mathrm{C}^{*}$-algebras. The study of sums and difference of idempotents has notably been studied in connections with the Drazin inverse (see for instance [12] and references therein). In this paper, we use the involutive structure of the ring and properties of the Moore-Penrose inverse to study particular idempotents, projections (self-adjoint idempotents).

It can be proved that for any $a \in \mathcal{R}$, there is at most one $a^{\dagger} \in \mathcal{R}$ such that

$$
a a^{\dagger} a=a, \quad a^{\dagger} a a^{\dagger}=a^{\dagger}, \quad\left(a a^{\dagger}\right)^{*}=a a^{\dagger}, \quad\left(a^{\dagger} a\right)^{*}=a^{\dagger} a .
$$

(see [3], [6], [7], [8], [16]). If there exists such $a^{\dagger}$ we will say that $a$ is MoorePenrose invertible and call $a^{\dagger}$ the Moore-Penrose inverse of $a$. The subset of 
$\mathcal{R}$ composed of all Moore-Penrose invertible elements will be denote by $\mathcal{R}^{\dagger}$. We write $\mathcal{R}^{-1}$ for the set of all invertible elements in $\mathcal{R}$.

We say that $a \in \mathcal{R}$ is relatively regular if there exists $b \in \mathcal{R}$ such that $a b a=a$. In this case $b$ is called an inner generalized inverse of $a$. A known result (see Theorem 1.4.11 of [3]) is the following: let $\mathcal{R}$ be a ring with involution obeying the Gelfand-Naimark property. Then $a \in \mathcal{R}$ is MoorePenrose invertible if and only if $a$ is relatively regular. Let us recall that a ring $\mathcal{R}$ with involution has the Gelfand-Naimark property if $1+x^{*} x \in \mathcal{R}^{-1}$ for all $x \in \mathcal{R}$. It is known that any $C^{*}$-algebra has the Gelfand-Naimark property. See also [6] and [11].

An element $a \in \mathcal{R}$ is left $*$-cancellable if $a^{*} a x=a^{*} a y$ implies $a x=a y$. Analogously, $a \in \mathcal{R}$ is right $*$-cancellable if $x a a^{*}=y a a^{*}$ implies $x a=y a$. Finally, $a \in \mathcal{R}$ is $*$-cancellable if it is both left and right $*$-cancellable. A ring $\mathcal{R}$ is called $*$-reducing if every element of $\mathcal{R}$ is $*$-cancellable. Let us remark that any $C^{*}$-algebra is a $*$-reducing ring.

We use the following notation: If $X, Y \subset \mathcal{R}$, then

$$
X \perp Y \quad \Longleftrightarrow \quad \forall(x, y) \in X \times Y, x^{*} y=0
$$

Observe that if $\mathcal{R}$ is $*$-reducing and if $X \neq \varnothing \neq Y$, then $X \perp Y$ implies $X \cap Y=\{0\}$.

Let $x \in \mathcal{R}$ and let $p \in \mathcal{R}$ be an idempotent $\left(p=p^{2}\right)$. Then we can write

$$
x=p x p+p x(1-p)+(1-p) x p+(1-p) x(1-p)
$$

and use the notations

$x_{11}=p x p, \quad x_{12}=p x(1-p), \quad x_{21}=(1-p) x p, \quad x_{22}=(1-p) x(1-p)$.

Every projection $p \in \mathcal{R}$ induces a matrix representation which preserves the involution in $\mathcal{R}$, namely $x \in \mathcal{R}$ can be represented by means of the following matrix:

$$
x=\left[\begin{array}{cc}
p x p & p x(1-p) \\
(1-p) x p & (1-p) x(1-p)
\end{array}\right]_{p}=\left[\begin{array}{ll}
x_{11} & x_{12} \\
x_{21} & x_{22}
\end{array}\right]_{p} .
$$


From now on, for an arbitrary projection $p$, we shall denote $\bar{p}=1-p$.

Two elementary facts derived from the definition of the Moore-Penrose inverse are the following:

(i) Let $a \in \mathcal{R}$. Then $a \in \mathcal{R}^{\dagger} \Longleftrightarrow a^{*} \in \mathcal{R}^{\dagger}$, and under this situation one has $\left(a^{*}\right)^{\dagger}=\left(a^{\dagger}\right)^{*}$.

(ii) Let $a \in \mathcal{R}^{\dagger}$. Then $a^{\dagger} \in \mathcal{R}^{\dagger}$ and $\left(a^{\dagger}\right)^{\dagger}=a$.

The following simple result also will be useful.

Lemma 2.1. Let $\mathcal{R}$ be a ring with involution and $a \in \mathcal{R}$. Then

(i) If $a \in \mathcal{R}^{\dagger}$, then $a^{*} a, a a^{*} \in \mathcal{R}^{\dagger}$ and

$\left(a^{*} a\right)^{\dagger}=a^{\dagger}\left(a^{*}\right)^{\dagger}, \quad\left(a a^{*}\right)^{\dagger}=\left(a^{*}\right)^{\dagger} a^{\dagger}, \quad a^{\dagger}=\left(a^{*} a\right)^{\dagger} a^{*}=a^{*}\left(a a^{*}\right)^{\dagger}, \quad a^{*}=a^{\dagger} a a^{*}=a^{*} a a^{\dagger}$.

(ii) If $\mathcal{R}$ is $*$-reducing, then $a^{*} a \in \mathcal{R}^{\dagger} \Rightarrow a \in \mathcal{R}^{\dagger}$ and $a a^{*} \in \mathcal{R}^{\dagger} \Rightarrow a \in \mathcal{R}^{\dagger}$.

Proof. The proof of (i) is a consequence of direct computations. We will prove only the first implication of (ii) since to prove the other one, it is sufficient to make the same argument for $a^{*}$ instead of $a$. Assume that $a^{*} a \in \mathcal{R}^{\dagger}$, and let $x=\left(a^{*} a\right)^{\dagger} a^{*}$. Observe that the Moore-Penrose inverse of a selfadjoint Moore-Penrose invertible element is again self-adjoint, and thus, $\left(a^{*} a\right)^{\dagger}$ is self-adjoint. Now $(a x)^{*}=\left[a\left(a^{*} a\right)^{\dagger} a^{*}\right]^{*}=a\left(a^{*} a\right)^{\dagger} a^{*}=a x$; $x a=\left(a^{*} a\right)^{\dagger} a^{*} a$ is selfadjoint; $x a x=\left(a^{*} a\right)^{\dagger} a^{*} a\left(a^{*} a\right)^{\dagger} a^{*}=\left(a^{*} a\right)^{\dagger} a^{*}=x$. Finally, $a^{*} a x a=a^{*} a\left(a^{*} a\right)^{\dagger} a^{*} a=a^{*} a$, and since $\mathcal{R}$ is $*$-reducing, we get $a x a=a$.

A simple consequence of Lemma 2.1 is the following: Let $x \in \mathcal{R}^{\dagger}$ be self-adjoint. Then $x x^{\dagger}=x^{\dagger} x$ and $x$ is the commuting (or group) inverse of $x$ (see for instance [6]). In fact, $x x^{\dagger}=x\left(x^{*} x\right)^{\dagger} x^{*}=x^{*}\left(x x^{*}\right)^{\dagger} x=x^{\dagger} x$. For a better insight on the the formulas of Lemma 2.1, commutation and cancellation properties, see [5]. For the class of elements $x$ in a $\mathrm{C}^{*}$-algebra such that $x x^{\dagger}=x^{\dagger} x$, the reader is reffered to [2, 10]. More generally, elements admitting both a group inverse and a Moore-Penrose inverse are discussed in [15] in the case of a ring. 
Let $p$ and $q$ be two projections in a ring $\mathcal{R}$ with involution. Then

$$
p=\left[\begin{array}{ll}
p & 0 \\
0 & 0
\end{array}\right]_{p} \quad \text { and } \quad q=\left[\begin{array}{cc}
a & b \\
b^{*} & d
\end{array}\right]_{p},
$$

where

$$
a=p q p, \quad b=p q(1-p), \quad d=(1-p) q(1-p) .
$$

Lemma 2.2. Let $p, q \in \mathcal{R}$ be projections given by (2.2). Then

(i) $a=a^{2}+b b^{*}$,

(ii) $b=a b+b d$,

(iii) $d=d^{2}+b^{*} b$,

Proof. All the equalities follow from the condition $q=q^{2}$.

The following result is a generalization of Lemma $3(\mathrm{v})-(\mathrm{x})$ from [1]:

Lemma 2.3. Let $\mathcal{R}$ be a $*$-reducing ring. If $p, q \in \mathcal{R}$ are projections given by (2.2), then the following hold:

(i) If $a$ is Moore-Penrose invertible, then $a a^{\dagger} b=b$,

(ii) If $1-a$ is Moore-Penrose invertible, then $(1-a)(1-a)^{\dagger} b=(p-a)(p-$ $a)^{\dagger} b=b$,

(iii) If $d$ is Moore-Penrose invertible, then $b d d^{\dagger}=b$,

(iv) If $1-d$ is Moore-Penrose invertible, then $b(1-d)(1-d)^{\dagger}=b(1-p-$ d) $(1-p-d)^{\dagger}=b$,

(v) If $a$ and $1-d$ are Moore-Penrose invertible, then $a^{\dagger} b=b(1-p-d)^{\dagger}=$ $b(1-d)^{\dagger}$,

(vi) If $1-a$ and $d$ are Moore-Penrose invertible, then $b d^{\dagger}=(1-a)^{\dagger} b=$ $(p-a)^{\dagger} b$. 
Proof. (i): Since $a=p q p=p q(p q)^{*}$ is Moore-Penrose invertible, by Lemma 2.1, we have that $p q$ is Moore-Penrose invertible and

$$
\begin{aligned}
a a^{\dagger} b & =p q(p q)^{*}\left(p q(p q)^{*}\right)^{\dagger} p q(1-p) \\
& =p q(p q)^{*}\left((p q)^{*}\right)^{\dagger}(p q)^{\dagger} p q(1-p) \\
& =p q(p q)^{\dagger} p q(p q)^{\dagger} p q(1-p) \\
& =b .
\end{aligned}
$$

To prove (ii), it is sufficient to use former item (i) for projections $p$ and $1-q$. If we use item (i) for projections $1-p$ and $q$, we get $d d^{\dagger} b^{*}=b^{*}$, and (iii) follows by taking $*$ in both sides. Item (iv) follows by using item (i) for projections $1-p$ and $1-q$.

(v): Observe that since $q$ is self-adjoint, then the representation of $q$ given in (2.2) implies that $a$ is self-adjoint, hence $a a^{\dagger}=a^{\dagger} a$. By condition (ii) of Lemma 2.2 and by (i), it follows that $a^{\dagger} b=a^{\dagger}(a b+b d)=a a^{\dagger} b+a^{\dagger} b d=$ $b+a^{\dagger} b d$. Hence,

$$
b=a^{\dagger} b(1-d)=a^{\dagger} b(1-p-d) .
$$

Multiplying the last equality from the left side by $(1-d)^{\dagger}$ and using (iv), we get that $b(1-d)^{\dagger}=a^{\dagger} b$. Similarly, $a^{\dagger} b=b(1-p-d)^{\dagger}$. The proof of (vi) follows by using item (v) for projections $p$ and $1-q$.

Lemma 2.4. Let $\mathcal{R}$ be a $*$-reducing ring. If $p, q \in \mathcal{R}$ are projections and $q$ is partitioned as in (2.2), then

(i) If $1-a$ and $d$ are Moore-Penrose invertible, then $a-b d^{\dagger} b^{*}=1-(1-$ a) $(1-a)^{\dagger}=p-(p-a)^{\dagger}(p-a)$,

(ii) If $a$ and $1-d$ are Moore-Penrose invertible, then $d-b^{*} a^{\dagger} b=1-(1-$ d) $(1-d)^{\dagger}=1-p-(1-p-d)(1-p-d)^{\dagger}$,

(iii) If $1-a$ and $d$ are Moore-Penrose invertible, then $d+b^{*}(1-a)^{\dagger} b=$ $d+b^{*}(p-a)^{\dagger} b=d d^{\dagger}$,

(iv) If $a$ and $1-d$ are Moore-Penrose invertible, then $a+b(1-d)^{\dagger} b^{*}=$ $a+b(1-p-d)^{\dagger} b^{*}=a a^{\dagger}$. 
Proof. (i): As we pointed out in the proof of item (v), Lemma 2.3, we have that $a$ is self-adjoint, hence $1-a$ is again self-adjoint, and thus, $(1-a)^{\dagger}(1-$ $a)=(1-a)(1-a)^{\dagger}$. Now we have

$$
1-a=(1-a)^{\dagger}(1-a)(1-a)=(1-a)^{\dagger}(1-a)-(1-a)^{\dagger}(1-a) a .
$$

By Lemma 2.3 (vi), Lemma 2.2 (i), and the previous computation, we get that $b d^{\dagger} b^{*}=(1-a)^{\dagger} b b^{*}=(1-a)^{\dagger}(1-a) a=(1-a)^{\dagger}(1-a)-(1-a)$. Hence, $a-b d^{\dagger} b^{*}=1-(1-a)(1-a)^{\dagger}$. The proofs of (ii)-(iv) are similar.

\section{Projections in rings with involution}

Theorem 3.1. Let $\mathcal{R}$ be a ring with involution and $p, q \in \mathcal{R}$ be projections. Then

$$
(p-q) \mathcal{R}=p \bar{q} \mathcal{R} \oplus^{\perp} \bar{p} q \mathcal{R} .
$$

Proof. It is evident that $p \bar{q} \mathcal{R} \perp \bar{p} q \mathcal{R}$. Now, we will prove that $(p-q) \mathcal{R}=$ $p \bar{q} \mathcal{R}+\bar{p} q \mathcal{R}$. Take any $z \in(p-q) \mathcal{R}$. We have that $z=(p-q) x$ for some $x \in \mathcal{R}$. If we take $y=(1-2 q) x$, we get $(p-q) x=p \bar{q} y+\bar{p} q y$, so $(p-q) \mathcal{R} \subseteq p \bar{q} \mathcal{R}+\bar{p} q \mathcal{R}$. For arbitrary $a \in p \bar{q} \mathcal{R}+\bar{p} q \mathcal{R}$, we have that $a=p \bar{q} y_{1}+\bar{p} q y_{1}$, for some $y_{1}, y_{2} \in \mathcal{R}$ and

$$
a=p \bar{q} y_{1}+\bar{p} q y_{2}=(p-q)\left(y_{1}-q y_{1}-q y_{2}\right)
$$

i.e., $a \in(p-q) \mathcal{R}$. Hence $p \bar{q} \mathcal{R}+\bar{p} q \mathcal{R} \subseteq(p-q) \mathcal{R}$. The proof is completed.

In [9, Th. 4.2] it was characterized when the difference of two projections is invertible. Former Theorem 3.1 permits give another characterization.

Corollary 3.1. Let $\mathcal{R}$ be a ring with involution and $p, q \in \mathcal{R}$ be projections. Then $p-q$ is invertible if and only if $p \bar{q} \mathcal{R} \oplus^{\perp} \bar{p} q \mathcal{R}=\mathcal{R}$.

Theorem 3.2. Let $\mathcal{R}$ be a ring with involution and $p, q \in \mathcal{R}$ be projections. The following statements are equivalent:

(i) $p+q \in \mathcal{R}^{-1}$, 
(ii) There exists $h \in \mathcal{R}$ such that $1=p h+q(1-h)$ and $(1-h) p=h q$,

(iii) There exists $h \in \mathcal{R}$ such that $h=p h, q(1-h)=1-h$ and $(1-h) p=h q$.

The element $h$ in conditions (ii) and (iii) is unique and it satisfies $h=$ $p(p+q)^{-1}$.

Proof. (i) $\Rightarrow$ (ii): Define $h=p(p+q)^{-1}$. Obviously, one has $(1-h) p=h q$. Since $(p+q) p=p+q+2 q p-q(p+q)$, we have $(p+q) h=1+2 q h-q$, which implies $1=p h+q(1-h)$.

(ii) $\Rightarrow$ (i): From $(1-h) p=h q$ we get $p=(p+q) h^{*}$. Now,

$$
(p+q)\left(1-h-h^{*}+2 h^{*} h\right)=p h+q(1-h)=1 .
$$

Since $p+q$ and $1-h-h^{*}+2 h^{*} h$ are self-adjoint, by taking $*$ in $(p+q)(1-$ $\left.h-h^{*}+2 h^{*} h\right)=1$ we get $\left(1-h-h^{*}+2 h^{*} h\right)(p+q)=1$.

(ii) $\Rightarrow$ (iii): Since we have proved (ii) $\Rightarrow$ (i), we get $p+q \in \mathcal{R}^{-1}$. The second condition of (ii) leads to $h=p(p+q)^{-1}$. Thus $p h=h$, and substituting this into $1=p h+q(1-h)$ leads to $1=h+q(1-h)$, i.e., $q(1-h)=1-h$.

(iii) $\Rightarrow$ (ii): It is evident.

Theorem 3.3. Let $\mathcal{R}$ be a ring with involution and $p, q \in \mathcal{R}$ be projections.

The following statements are equivalent:

(i) There exists $h \in \mathcal{R}$ such that $h=p h$ and $q(1-h)=1-h$,

(ii) $\mathcal{R}=p \mathcal{R}+q \mathcal{R}$.

Proof. (i) $\Rightarrow$ (ii) follows from $1=h+(1-h)=p h+q(1-h) \in p \mathcal{R}+q \mathcal{R}$.

(ii) $\Rightarrow$ (i): Let $x, y \in \mathcal{R}$ be such that $1=p x+q y$ and let us denote $h=p x$. Now, $p h=h$ and $q(1-h)=q(1-p x)=q q y=q y=1-p x=1-h$.

As a corollary of Theorem 3.2, Theorem 3.3 and [9, Theorem 4.4], we have the following:

Corollary 3.2. Let $\mathcal{R}$ be a ring with involution and $p, q \in \mathcal{R}$ be projections. The following statements are equivalent: 
1) $p+q \in \mathcal{R}^{-1}$ and $h=p(p+q)^{-1}$ is idempotent,

2) $\mathcal{R}=p \mathcal{R} \oplus q \mathcal{R}$,

3) $p-q \in \mathcal{R}^{-1}$

4) There exists idempotent $k$ such that $k \mathcal{R}=p \mathcal{R}$ and $(1-k) \mathcal{R}=q \mathcal{R}$.

Proof. 1) $\Rightarrow 2) h=p(p+q)^{-1} \Rightarrow(1-h)=q(p+q)^{-1}$ and $h \mathcal{R}=p \mathcal{R}$, $(1-h) \mathcal{R}=q \mathcal{R} . h^{2}=h$ then implies $\mathcal{R}=h \mathcal{R} \oplus(1-h) \mathcal{R}=p \mathcal{R} \oplus q \mathcal{R}$.

2) $\Rightarrow 3) \Rightarrow 4$ ) by $[9$, Theorem 4.4].

$4) \Rightarrow 1)$ By Lemma 4.1, we have that $k$ satisfies $p k=k, k p=p$, $q(1-k)=k$ and $(1-k) q=q$. It follows that $(1-k) p=0=k q$ and $k$ satisfies condition $i i i)$ of Theorem 3.2.

\section{$4 \quad$ Projections in $*$-reducing rings}

We shall need the following simple lemma:

Lemma 4.1. Let $x, y \in \mathcal{R}$.

1) If $x, y$ and $x y$ are self-adjoint, then $y x=x y$.

2) Selfadjoint $x$ is an invertible if and only if there exists $y \in \mathcal{R}$ such that $x y=1$ if and only if $x \mathcal{R}=\mathcal{R}$.

3) If $e$ and $f$ are idempotents, then $e \mathcal{R}=f \mathcal{R}$ if and only if ef $=f$ and $f e=e$.

4) If $p, q$ are projectors and $p \mathcal{R}=q \mathcal{R}$, then $p=q$.

Proof. The proofs of 1) and 2) are trivial.

3) Let $e, f \in \mathcal{R}$ be two idempotents such that $e \mathcal{R}=f \mathcal{R}$. Since $e \in e \mathcal{R}=$ $f \mathcal{R}$, then exists $t \in \mathcal{R}$ such that $e=f t$, so $f e=e$. By reversing the roles of $e$ and $f$ we have $e f=f$.

4) It follows by 3 ). 
The following result which will be of major importance in the sequel, give sufficient conditions for the Moore-Penrose invertibility of several elements in a $*$-reducing ring of the form $f(p, q)$, where $p$ and $q$ are two projections and $f$ is a polynomial in two non-commuting variables.

Theorem 4.1. Let $\mathcal{R}$ be $a *$-reducing ring. If $p, q \in \mathcal{R}$ are projections, then

(i) If $p \bar{q} p$ and $\bar{p} q \bar{p}$ are Moore-Penrose invertible, then $p+q$ is MoorePenrose invertible and

$$
(p+q)(p+q)^{\dagger}=p+\bar{p} q(\bar{p} q \bar{p})^{\dagger} .
$$

(ii) If $\bar{p} q \bar{p}$ is Moore-Penrose invertible, then $\bar{p} q$ is Moore-Penrose invertible and

$$
(\bar{p} q)^{\dagger}=q(\bar{p} q \bar{p})^{\dagger}
$$

(iii) If $p \bar{q} p$ and $\bar{p} q \bar{p}$ are Moore-Penrose invertible, then $p-q$ is MoorePenrose invertible and

$$
(p-q)(p-q)^{\dagger}=(p-p q p)(p-p q p)^{\dagger}+\bar{p} q(\bar{p} q \bar{p})^{\dagger} .
$$

(iv) If $p q \bar{p}$ is Moore-Penrose invertible, then $p q-q p$ is Moore-Penrose invertible and

$$
(p q-q p)(p q-q p)^{\dagger}=p q(p q \bar{p})^{\dagger}+(p q \bar{p})^{\dagger} q \bar{p} .
$$

Proof. (i): Let us suppose that the projections $p$ and $q$ are represented as in (2.2). By hypothesis one has that $p-a, d \in \mathcal{R}^{\dagger}$. Since $1-a=(p-a)+(1-p)$ and $p-a, \bar{p} \in \mathcal{R}^{\dagger}$ (observe that since $\bar{p}$ is a projection, obviously $\bar{p} \in \mathcal{R}^{\dagger}$ and $\left.\bar{p}^{\dagger}=\bar{p}\right)$ we get $1-a \in \mathcal{R}^{\dagger}$. Let

$$
x=\frac{1}{2}\left(p+(p-a)(p-a)^{\dagger}\right)-b d^{\dagger}-d^{\dagger} b^{*}+2 d^{\dagger}-d d^{\dagger} .
$$

We shall prove that $x=(p+q)^{\dagger}$ by verifying the four conditions of the Moore-Penrose invertibility. We shall decompose $x$ as in (2.1). Obviously we have

$p x=x_{11}+x_{12} \quad$ and $\quad q x=a x_{11}+b x_{21}+a x_{12}+b x_{22}+b^{*} x_{11}+d x_{21}+b^{*} x_{12}+d x_{22}$, 
where

$x_{11}=\frac{1}{2}\left(p+(p-a)(p-a)^{\dagger}\right), \quad x_{12}=-b d^{\dagger}, \quad x_{21}=-d^{\dagger} b^{*}, \quad x_{22}=2 d^{\dagger}-d d^{\dagger}$.

Let us remark that $p-a$ is self-adjoint. Then

$$
p-a=(p-a)(p-a)(p-a)^{\dagger}=(p-a)(p-a)^{\dagger}-a(p-a)(p-a)^{\dagger},
$$

and thus, by utilizing Lemma 2.4 (i) we get

$$
\begin{aligned}
(p+a)\left(p+(p-a)(p-a)^{\dagger}\right) & =p+(p-a)(p-a)^{\dagger}+a+a(p-a)(p-a)^{\dagger} \\
& =2\left[(p-a)(p-a)^{\dagger}+a\right] \\
& =2\left[p+b d^{\dagger} b^{*}\right] .
\end{aligned}
$$

Thus,

$$
x_{11}+a x_{11}+b x_{21}=\frac{1}{2}(p+a)\left(p+(p-a)(p-a)^{\dagger}\right)-b d^{\dagger} b^{*}=p .
$$

Observe that Lemma 2.3 (ii) in conjunction with Lemma 2.3 (vi) can be written $b d^{\dagger}-b=a b d^{\dagger}$. Hence by Lemma 2.3 (iii), we get

$$
x_{12}+a x_{12}+b x_{22}=-b d^{\dagger}-a b d^{\dagger}+b\left(2 d^{\dagger}-d d^{\dagger}\right)=0 .
$$

Lemma 2.3 (ii) and the self-adjointness of $a$ imply $b^{*}(p-a)(p-a)^{\dagger}=b^{*}$. Furthermore, from the definition of $b$ given in (2.3) we trivially get $b^{*} p=b$. Now, Lemma 2.3 (iii) yields

$$
b^{*} x_{11}+d x_{21}=\frac{1}{2} b^{*}\left(p+(p-a)(p-a)^{\dagger}\right)-d d^{\dagger} b^{*}=0 .
$$

Since $q$ is self-adjoint, the representation of $q$ given in (2.2) yields that $d$ is self-adjoint, hence $d d^{\dagger}=d^{\dagger} d$. In view of 2.2 (iii), we have

$$
b^{*} x_{12}+d x_{22}=\left(d^{2}-d\right) d^{\dagger}+2 d d^{\dagger}-d=d d^{\dagger} .
$$

The above computations show that

$$
(p+q) x=p+d d^{\dagger} .
$$


Thus, $(p+q) x$ is self-adjoint. Since $x, p+q$, and $(p+q) x$ are self-adjoint, fact (??) permits get that $x(p+q)=(p+q) x$. By Lemma 2.3 (iii) and (4.2) we easily have $(p+q) x(p+q)=p+q$ and $x(p+q) x=x$.

Now, since $d=(1-p) q(1-p)$, it is evident that (i) holds.

(ii): Since $(\bar{p} q)(\bar{p} q)^{*}=\bar{p} q \bar{p} \in \mathcal{R}^{\dagger}$, by Lemma 2.1 we have $\bar{p} q \in \mathcal{R}^{\dagger}$ and

$$
(\bar{p} q)^{\dagger}=(\bar{p} q)^{*}\left(\bar{p} q(\bar{p} q)^{*}\right)^{\dagger}=q \bar{p}(\bar{p} q \bar{p})^{\dagger}=q(\bar{p} q \bar{p})^{\dagger} .
$$

By computation we get that $\bar{p} q(\bar{p} q)^{\dagger}=\bar{p} q \bar{p}(\bar{p} q \bar{p})^{\dagger}$. Now $(\bar{p} q)^{\dagger} \bar{p} q$ is selfadjoint, hence

$$
(\bar{p} q)^{\dagger} \bar{p} q=\left[(\bar{p} q)^{\dagger} \bar{p} q\right]^{*}=q \bar{p}\left[(\bar{p} q)^{\dagger}\right]^{*}=q \bar{p}\left[(\bar{p} q)^{*}\right]^{\dagger}=q \bar{p}(q \bar{p})^{\dagger},
$$

and thus, by Lemma 2.1,

$\bar{p} q=\bar{p} q(\bar{p} q)^{\dagger} \bar{p} q=\bar{p} q \bar{p}(q \bar{p})^{\dagger}=\bar{p} q \bar{p}(q \bar{p})^{*}\left[q \bar{p}(q \bar{p})^{*}\right]^{\dagger}=\bar{p} q(q \bar{p})^{*}\left[q \bar{p}(q \bar{p})^{*}\right]^{\dagger}=\bar{p} q(q \bar{p})^{\dagger}$.

(iii): Let us denote $z=\left[\begin{array}{cc}(p-a)(p-a)^{\dagger} & -b d^{\dagger} \\ -d^{\dagger} b^{*} & -d d^{\dagger}\end{array}\right]_{p}$. By a direct computation and Lemma 2.2 (iii), Lemma 2.3 (ii), (iii), (vi), and Lemma 2.4 (i) we get $(p-q) z=(p-a)(p-a)^{\dagger}+d d^{\dagger}$ is self-adjoint. Since $z$ is self-adjoint we get that $z(p-q)=(p-q) z$. By Lemma 2.3 (ii), (iii) we get $(p-q) z(p-q)=p-q$ and $z(p-q) z=z$. Thus, $p-q \in \mathcal{R}^{\dagger}$ and $z=(p-q)^{\dagger}$.

(iv): Observe that $p q-q p=b-b^{*}$. Since $b=p q \bar{p} \in \mathcal{R}^{\dagger}$ and $b^{\dagger}=$ $b^{*}\left(b b^{*}\right)^{\dagger} \in \bar{p} \mathcal{R} p$, by a direct verification of the four Moore-Penrose equations and using Lemma 2.1 we get $(p q-q p)^{\dagger}=b^{\dagger}-\left(b^{*}\right)^{\dagger}$ and $(p q-q p)(p q-q p)^{\dagger}=$ $b b^{\dagger}+b^{\dagger} b$.

Corollary 4.1. Let $\mathcal{R}$ be a *-reducing ring. If $p, q \in \mathcal{R}$ are projections such that pqp is Moore-Penrose invertible, then $p q$ is also Moore-Penrose invertible and $(p q)^{\dagger}=q(p q p)^{\dagger}$.

Proof. It follows from Lemma 4.1 (ii) by changing $p$ by $\bar{p}$.

The following result is a generalization of the result given in $[14, \mathrm{Th} .3$, Th. 4] for the matrix case:

Lemma 4.2. Let $\mathcal{R}$ be a *-reducing ring. and let $p, q \in \mathcal{R}$ be projections such that $p \bar{q} p$ and $\bar{p} q \bar{p}$ are Moore-Penrose invertible. Then 
(i) $x=p+\bar{p}(\bar{p} q)^{\dagger}$ is a projection and $x \mathcal{R}=p \mathcal{R}+q \mathcal{R}$,

(ii) $y=p-p(p \bar{q})^{\dagger}$ is a projection and $y \mathcal{R}=p \mathcal{R} \cap q \mathcal{R}$.

Proof. (i): By Theorem 4.1 (i) and (ii) we have $x=p+\bar{p}(\bar{p} q)^{\dagger}=p+$ $\bar{p} q(\bar{p} q \bar{p})^{\dagger}=(p+q)(p+q)^{\dagger}$, which implies that $x$ is a projection. Since, $x=p(p+q)^{\dagger}+q(p+q)^{\dagger}$, it is evident that $x \mathcal{R} \subseteq p \mathcal{R}+q \mathcal{R}$. Observe that $\bar{p} q(\bar{p} q)^{\dagger}=\left[\bar{p} q(\bar{p} q)^{\dagger}\right]^{*}=\left[(\bar{p} q)^{\dagger}\right]^{*} q \bar{p}$, which yields $x p=\left(p+\bar{p} q(\bar{p} q)^{\dagger}\right) p=p$. from $p+q=(p+q)(p+q)^{\dagger}(p+q)=x(p+q)=x p+x q$ and $x p=x$ we deduce $x q=q$. Finally, $p \mathcal{R}+q \mathcal{R}=x p \mathcal{R}+x q \mathcal{R} \subseteq x \mathcal{R}$.

(ii): First of all, we must prove that the definition of $y$ is meaningful, in other words, we must prove that $p \bar{q} \in \mathcal{R}^{\dagger}$; but this follows easily from $p-p q p \in \mathcal{R}^{\dagger},(p \bar{q})(p \bar{q})^{*}=p \bar{q} p=p-p q p$ and Lemma 2.1. Since $y=$ $p-p \bar{q}(p \bar{q})^{\dagger}$, we have that $y$ is self-adjoint. Using that $(p \bar{q})^{\dagger} p=(p \bar{q})^{\dagger}$, we trivially get $y^{2}=y$. Let us remark that we can write $y=p-p \bar{q}(p \bar{q})^{\dagger} p$. Evidently, $y \in p \mathcal{R}$, so $y \mathcal{R} \subseteq p \mathcal{R}$. Since $y \bar{q}=\left(p-p \bar{q}(p \bar{q})^{\dagger} p\right) \bar{q}=0$, we have $y q=y$. By taking $*$ in the last equality we have $q y=y$, hence $y \mathcal{R} \subseteq q \mathcal{R}$. Thus $y \mathcal{R} \subseteq p \mathcal{R} \cap q \mathcal{R}$.

To prove $p \mathcal{R} \cap q \mathcal{R} \subseteq y \mathcal{R}$, take arbitrary $z \in p \mathcal{R} \cap q \mathcal{R}$. Observe that

$$
p \bar{q}(p \bar{q})^{\dagger}=\left[p \bar{q}(p \bar{q})^{\dagger}\right]^{*}=\left[(p \bar{q})^{\dagger}\right]^{*} \bar{q} p=\left[(p \bar{q})^{*}\right]^{\dagger} \bar{q} p=(\bar{q} p)^{\dagger} \bar{q} p .
$$

Since $z=p z=q z$, it follows that $y z=\left(p-(\bar{q} p)^{\dagger} \bar{q} p\right) z=z$. Hence, $p \mathcal{R} \cap q \mathcal{R} \subseteq$ $y \mathcal{R}$.

Next four results continue the study of the sum $p+q$ and the ideals $(p+q) \mathcal{R},(p+q)^{\circ}$ under the additional assumption that $p \bar{q} p$ and $\bar{p} q \bar{p}$ are Moore-Penrose invertible.

Theorem 4.2. Let $\mathcal{R}$ be $a *$-reducing ring and $p, q \in \mathcal{R}$ be two projections such that $p \bar{q} p$ and $\bar{p} q \bar{p}$ are Moore-Penrose invertible. Then

(i) $(p+q) \mathcal{R}=\bar{p} q \bar{p} \mathcal{R} \oplus^{\perp} p \mathcal{R}$.

(ii) $(p+q) \mathcal{R}=\bar{p} q \mathcal{R} \oplus^{\perp} p \mathcal{R}$.

(iii) $(p+q) \mathcal{R}=p \mathcal{R}+q \mathcal{R}$. 
(iv) $(p+q) \mathcal{R}=(p-q) \mathcal{R} \oplus^{\perp}(p \mathcal{R} \cap q \mathcal{R})$.

(v) $(p+q)^{\circ}=(\bar{p} q)^{\circ} \cap p^{\circ}$.

(vi) $(p+q)^{\circ}=p^{\circ} \cap q^{\circ}$.

Proof. (i): It is evident that $\bar{p} q \bar{p} \mathcal{R} \perp p \mathcal{R}$. By Lemma 2.1 (i), we have that

$$
p+q=\bar{p} q+p(1+q)=\bar{p} q(\bar{p} q)^{\dagger} \bar{p} q+p(1+q)=\bar{p} q \bar{p}(\bar{p} q \bar{p})^{\dagger} \bar{p} q+p(1+q),
$$

which implies that $(p+q) \mathcal{R} \subseteq \bar{p} q \bar{p} \mathcal{R}+p \mathcal{R}$. To prove the opposite inclusion, take arbitrary $z \in \bar{p} q \bar{p} \mathcal{R}+p \mathcal{R}$. We have $z=\bar{p} q \bar{p} x+p y$ for some $x, y \in \mathcal{R}$. By Theorem 4.1 (i), we get $(p+q)(p+q)^{\dagger} z=z$, so $z \in(p+q) \mathcal{R}$. Hence $(p+q) \mathcal{R}=\bar{p} q \bar{p} \mathcal{R}+p \mathcal{R}$.

(ii): Since for any Moore-Penrose invertible $a \in \mathcal{R}$, one has $a \mathcal{R}=a a^{\dagger} \mathcal{R}$, by Theorem 4.1 (ii), we have $\bar{p} q \mathcal{R}=\bar{p} q \bar{p} \mathcal{R}$. Now, the assertation follows by item (i).

(iii): The inclusion $\subseteq$ is evident. To prove the opposite, let us use the notations given in (2.3) and let us demonstrate $p q+d d^{\dagger} q=q$. In fact, Lemma 2.3 (iii) yields $d d^{\dagger} b^{*}=b^{*}$ and now

$$
p q+d d^{\dagger} q=p q+d d^{\dagger}(1-p) q=p q+d d^{\dagger}\left(b^{*}+d\right)=p q+b+d=q .
$$

If $x \in p \mathcal{R}+q \mathcal{R}$, then exist $u, v \in \mathcal{R}$ such that $x=p u+q v$. By Theorem 4.1 (i), one gets

$$
(p+q)(p+q)^{\dagger} x=\left(p+d d^{\dagger}\right)(p u+q v)=p u+p q v+d d^{\dagger} q v=p u+q v=x .
$$

Thus, $x=(p+q)(p+q)^{\dagger} x \in(p+q) \mathcal{R}$.

(iv): By Theorem 4.1 (i) and (iii) and (2.3), it follows that $(p+q)(p+q)^{\dagger}=$ $p+d d^{\dagger}$ and $(p-q)(p-q)^{\dagger}=(p-a)(p-a)^{\dagger}+d d^{\dagger}$. By Lemma 4.2 (ii), it follows that $y=p-p(p \bar{q})^{\dagger}$ is a projection and $y \mathcal{R}=p \mathcal{R} \cap q \mathcal{R}$. If in Theorem 4.1 (ii), we replace $p$ with $\bar{p}$ and $q$ with $\bar{q}$, we get that $(p \bar{q})^{\dagger}=\bar{q}(p \bar{q} p)^{\dagger}$, so $y=p-(p-a)(p-a)^{\dagger}$. Now, since $p-(p-a)(p-a)^{\dagger}$ and $(p-a)(p-a)^{\dagger}+d d^{\dagger}$ 
are commuting projections which product is equal to zero, we have

$$
\begin{aligned}
(p+q) \mathcal{R}=\left(p+d d^{\dagger}\right) \mathcal{R} & =\left(\left((p-a)(p-a)^{\dagger}+d d^{\dagger}\right)+\left(p-(p-a)(p-a)^{\dagger}\right)\right) \mathcal{R} \\
& =\left((p-a)(p-a)^{\dagger}+d d^{\dagger}\right) \mathcal{R} \oplus^{\perp}\left(p-(p-a)(p-a)^{\dagger}\right) \mathcal{R} \\
& =(p-q) \mathcal{R} \oplus^{\perp}(p \mathcal{R} \cap q \mathcal{R}) .
\end{aligned}
$$

( $\subseteq \subseteq$ ): Let $x \in(p+q)^{\circ}$. By Theorem 4.1 (i) and by employing the notations given in 2.2 we have $0=(p+q)^{\dagger}(p+q) x=\left(p+d d^{\dagger}\right) x=p x+d d^{\dagger} x$; which by premultiplying by $p$ and $\bar{p}$ we get $0=p x$ and $0=d d^{\dagger} x$, respectively. Notice that $0=d d^{\dagger} x \Longleftrightarrow d x=0$. Finally, $\bar{p} q x=\left(b^{*}+d\right) x=b^{*} x+d x=$ $\bar{p} q p x+d x=0$.

$(\mathrm{v} \supseteq)$ : Let $x \in(\bar{p} q)^{\circ} \cap p^{\circ}$. we have $0=\bar{p} q x=\left(b^{*}+d\right) x=(\bar{p} q p+d) x=d x$. From Theorem 4.1 (i) we get $(p+q)(p+q)^{\dagger} x=\left(p+d d^{\dagger}\right) x=p x+d d^{\dagger} x=0$, which leads to $(p+q) x=0$.

(vi): The inclusion $p^{\circ} \cap q^{\circ} \subseteq(p+q)^{\circ}$ is obvious. Let $x \in(p+q)^{\circ}$. As in the proof of $(\mathrm{i} \subseteq)$ we get $p x=0$. Now, $(p+q) x=0$ leads to $q x=0$.

Observe that Theorem 4.2 (ii) and (vi) generalize to [14, Cor. 2]. Remark that Theorem 6 and Theorem 8 from [1] are equivalent (replacing $P$ with $I-Q$ and $Q$ with $P$ in Theorem 6, we get Theorem 8). The following corollary follows immediately from Theorem 4.2 .

Corollary 4.2. Let $\mathcal{R}$ be $a *$-reducing ring and $p, q \in \mathcal{R}$ be two projections such that $p \bar{q} p$ and $\bar{p} q \bar{p}$ are Moore-Penrose invertible. Then the following statements are equivalent:

(i) $p+q$ is invertible,

(ii) $\mathcal{R}=\bar{p} q \mathcal{R} \oplus^{\perp} p \mathcal{R}$,

(iii) $\mathcal{R}=\bar{p} q \bar{p} \mathcal{R} \oplus^{\perp} p \mathcal{R}$,

(iv) $\mathcal{R}=p \mathcal{R}+q \mathcal{R}$.

Theorem 4.3. Let $\mathcal{R}$ be $a *$-reducing ring and $p, q \in \mathcal{R}$ be two projections such that $\bar{p} q \bar{p}$ and $p \bar{q} p$ are Moore-Penrose invertible. Then 
(i) $(1-\bar{p} \bar{q}) \mathcal{R}=(p+q) \mathcal{R}$,

(ii) $(1-\bar{p} \bar{q})^{\circ}=(p+q)^{\circ}$.

Proof. (i): By Theorem 4.1 (i), using the notations given by (2.3), we get that $(p+q)(p+q)^{\dagger}=p+d d^{\dagger}$. On the other side, since $1-\bar{p} \bar{q}=p+b^{*}+d$, by Lemma 2.3 it is easy to check that $(1-\bar{p} \bar{q})^{\dagger}=d^{\dagger}-b^{*}(p-a)^{\dagger}+p$, so $(1-\bar{p} \bar{q})(1-\bar{p} \bar{q})^{\dagger}=p+d d^{\dagger}$. Hence, $(1-\bar{p} \bar{q})(1-\bar{p} \bar{q})^{\dagger}=(p+q)(p+q)^{\dagger}$ which is equivalent to $(1-\bar{p} \bar{q}) \mathcal{R}=(p+q) \mathcal{R}$.

(ii): By Theorem $4.2(\mathrm{v})$, we have $(p+q)^{\circ}=(\bar{p} q)^{\circ} \cap p^{\circ}$. Let us prove $(1-\bar{p} \bar{q})^{\circ}=(\bar{p} q)^{\circ} \cap p^{\circ}$ : If $x \in(1-\bar{p} \bar{q})^{\circ}$, then $\bar{p} \bar{q} x=x$, which yields $p q x=p x+q x$, hence $p x=0$ and $(1-p) q x=0$, in other words, $x \in(\bar{p} q)^{\circ} \cap p^{\circ}$. The opposite inclusion is evident.

Theorem 4.4. Let $\mathcal{R}$ be $a *$-reducing ring and $p, q \in \mathcal{R}$ be two projections such that $p \bar{q} p$ and $\bar{p} q \bar{p}$ are Moore-Penrose invertible. Then

(i) $(p q-q p) \mathcal{R} \subseteq(p+q) \mathcal{R}$.

(ii) $(p+q)^{\circ} \subseteq(p q-q p)^{\circ}$.

Proof. (i): By Theorem 4.1 (i), we have that $(p+q)(p+q)^{\dagger}=p+\bar{p} q(\bar{p} q \bar{p})^{\dagger}$. Now, by Lemma 2.3 (iii) using the notations from (2.3), we have that

$$
\begin{aligned}
(p+q)(p+q)^{\dagger}(p q-q p) & =\left(p+\bar{p} q(\bar{p} q \bar{p})^{\dagger}\right)(p q-q p) \\
& =p q-p q p-\bar{p} q(\bar{p} q \bar{p})^{\dagger} \bar{p} q p \\
& =p q(1-p)-\bar{p} q \bar{p}(\bar{p} q \bar{p})^{\dagger} \bar{p} q p \\
& =b-d d^{\dagger} b^{*} \\
& =b-b^{*} \\
& =p q-q p
\end{aligned}
$$

which implies that $(p q-q p) \mathcal{R} \subseteq(p+q) \mathcal{R}$.

(ii): It follows from Theorem 4.2 (vi)

Note that the inclusions in the previous theorem can be strict, as shows the case $q=1-p$. Next theorems study further the image ideal $(p q-q p) \mathcal{R}$ together with the ideals $(p-q) \mathcal{R}$ and $(1-p-q) \mathcal{R}$. 
Theorem 4.5. Let $\mathcal{R}$ be $a$ *-reducing ring and $p, q \in \mathcal{R}$ be two projections such that $\bar{p} \bar{q} \bar{p}, p \bar{q} p, p q p, \bar{p} q \bar{p}$ and $p q \bar{p}$ are Moore-Penrose invertible. Then

(i) $(p-q)(p-q)^{\dagger}+(1-p-q)(1-p-q)^{\dagger}-(p q-q p)(p q-q p)^{\dagger}=1$,

(ii) $(p-q) \mathcal{R}+(1-p-q) \mathcal{R}=\mathcal{R}$,

(iii) $(p-q)(p-q)^{\dagger}(1-p-q)(1-p-q)^{\dagger}=(p q-q p)(p q-q p)^{\dagger}$.

Proof. (i): Let $x=(p-q)(p-q)^{\dagger}+(1-p-q)(1-p-q)^{\dagger}-(p q-q p)(p q-q p)^{\dagger}$. By the proof of Theorem 4.10 we have $(p-q)(p-q)^{\dagger}=(p-a)(p-a)^{\dagger}+d d^{\dagger}$. By doing the same computation having replaced $p$ by $\bar{p}$ we get $(1-p-$ $q)(1-p-q)^{\dagger}=(1-p-d)(1-p-d)^{\dagger}+a a^{\dagger}$. By Theorem 4.1 (iv) we get $(p q-q p)(p q-q p)^{\dagger}=b b^{\dagger}+b^{\dagger} b$. Thus,

$$
x=(p-a)(p-a)^{\dagger}+d d^{\dagger}+(1-p-d)(1-p-d)^{\dagger}+a a^{\dagger}-b b^{\dagger}-b^{\dagger} b .
$$

Denote $y=a a^{\dagger}+(p-a)(p-a)^{\dagger}-b b^{\dagger}$. Since $a$ is self-adjoint, we get that the elements of the set $\left\{a, a^{\dagger}, p-a,(p-a)^{\dagger}\right\}$ are mutually commuting by Lemma 4.1. Now it is easy to prove by the definition of the Moore-Penrose inverse that $[a(p-a)]^{\dagger}=a^{\dagger}(p-a)^{\dagger}$. By Lemma 2.2 (i), we have

$b b^{\dagger}=b b^{*}\left(b b^{*}\right)^{\dagger}=\left(a-a^{2}\right)\left(a-a^{2}\right)^{\dagger}=a(p-a)[a(p-a)]^{\dagger}=a a^{\dagger}(p-a)(p-a)^{\dagger}$

so $(p-a)(p-a)^{\dagger}-b b^{\dagger}=\left(1-a a^{\dagger}\right)(p-a)(p-a)^{\dagger}$. Using that $\left(1-a a^{\dagger}\right)(p-$ $a)(p-a)^{\dagger}$ is a self-adjoint idempotent we get

$$
\begin{aligned}
y & =a a^{\dagger}+\left(1-a a^{\dagger}\right)(p-a)(p-a)^{\dagger} \\
& =a a^{\dagger}+\left(1-a a^{\dagger}\right)\left(\left(1-a a^{\dagger}\right)(p-a)(p-a)^{\dagger}\right) \\
& =a a^{\dagger}+\left(1-a a^{\dagger}\right)\left(\left(1-a a^{\dagger}\right)(p-a)(p-a)^{\dagger}\right)^{\dagger} .
\end{aligned}
$$

By Lemma $4.2(\mathrm{i})$, it follows that $y$ is a projection and $y \mathcal{R}=a a^{\dagger} \mathcal{R}+(p-$ $a)(p-a)^{\dagger} \mathcal{R}=a \mathcal{R}+(p-a) \mathcal{R}=p \mathcal{R}$. So, $y=p$. Similarly, we get that

$$
d d^{\dagger}+(1-p-d)(1-p-d)^{\dagger}-b^{\dagger} b=1-p .
$$

Hence $x=1$. 
(ii): It follows from Theorem 3.1.

(iii): By the proof of the item (i) of this Theorem we have that

$$
\begin{aligned}
(p-q) & (p-q)^{\dagger}(1-p-q)(1-p-q)^{\dagger} \\
& =\left((p-a)(p-a)^{\dagger}+d d^{\dagger}\right)\left((1-p-d)(1-p-d)^{\dagger}+a a^{\dagger}\right) \\
& =(p-a)(p-a)^{\dagger} a a^{\dagger}+d d^{\dagger}(1-p-d)(1-p-d)^{\dagger} .
\end{aligned}
$$

As in (i), we have that $b b^{\dagger}=a a^{\dagger}(p-a)(p-a)^{\dagger}$ and similarly $b^{\dagger} b=d d^{\dagger}(1-p-$ $d)(1-p-d)^{\dagger}$. Again, the proof of (i) distills $(p q-q p)(p q-q p)^{\dagger}=b b^{\dagger}+b^{\dagger} b$. The proof is completed.

Theorem 4.6. Let $\mathcal{R}$ be $a *$-reducing ring and $p, q \in \mathcal{R}$ be two projections such that $p q p, p \bar{q} p, \bar{p} q \bar{p}$ and $\bar{p} \bar{q} \bar{p}$ are Moore-Penrose invertible. The following conditions are equivalent:

(i) $(p q-q p) \mathcal{R}=(p-q) \mathcal{R}$,

(ii) $(1-p-q) \mathcal{R}=\mathcal{R}$,

(iii) $p=p q(p q)^{\dagger}, q=q p(q p)^{\dagger}$.

Proof. (i) $\Leftrightarrow$ (ii): By Theorem 4.1 (iii) and (iv) using the notations given in (2.3), we have that (i) is equivalent to

$$
(p-a)(p-a)^{\dagger}=b b^{\dagger} \text { and } d d^{\dagger}=b^{\dagger} b .
$$

Similarly, $(1-p-q) \mathcal{R}=\mathcal{R}$ is equivalent to $(1-p-q)(1-p-q)^{\dagger}=1$. If in Theorem 4.1 (iii) we replace $p$ with $\bar{p}$, we have that $(1-p-q)(1-p-q)^{\dagger}=$ $(1-p-d)(1-p-d)^{\dagger}+a a^{\dagger}$. Hence, (ii) is equivalent to $(1-p-d)(1-p-$ $d)^{\dagger}+a a^{\dagger}=1$, i.e.

$$
(1-p-d)(1-p-d)^{\dagger}=1-p \text { and } a a^{\dagger}=p .
$$

By (4.3), we have that $b b^{\dagger}=a a^{\dagger}(p-a)(p-a)^{\dagger}$. Now, multiplying the equality given in Lemma 2.4 (i) by $a a^{\dagger}$ from the left side and using Lemma 2.3 (i), we get that

$$
p-(p-a)(p-a)^{\dagger}=a a^{\dagger}-a a^{\dagger}(p-a)(p-a)^{\dagger} .
$$


Now, it is evident that $(p-a)(p-a)^{\dagger}=b b^{\dagger}$ if and only if $a a^{\dagger}=p$. Analogously, using (4.4), we get that $d d^{\dagger}=b^{\dagger} b$ if and only if $(1-p-d)(1-p-d)^{\dagger}=$ $1-p$. Hence, (i) and (ii) are equivalent.

(ii) $\Leftrightarrow$ (iii): By Corollary 4.1 and Lemma 2.3 (i), we have that (iii) is equivalent to $p=p q(p q)^{\dagger}=p q(p q p)^{\dagger}=a a^{\dagger}$ and $q=q p(q p)^{\dagger}=(p q)^{\dagger} p q=$ $q p(p q p)^{\dagger} p q=a+b+b^{*}+b^{*} a^{\dagger} b$. Now, by Lemma 2.3 (ii), we get that (iii) is equivalent to $p=a a^{\dagger}$ and $(1-p-d)(1-p-d)^{\dagger}=1-p$, which together with (4.6) implies that (ii) and (iii) are equivalent.

Theorem 4.7. Let $\mathcal{R}$ be $a *$-reducing ring and $p, q \in \mathcal{R}$ be two projections such that $p \bar{q} p, \bar{p} q \bar{p}, p q \bar{p}, \bar{p} \bar{q} \bar{p}$ and $p q p$ are Moore-Penrose invertible. The following conditions are equivalent:

(i) $(p q-q p) \mathcal{R}=\mathcal{R}$,

(ii) $(p-q) \mathcal{R}=\mathcal{R},(1-p-q) \mathcal{R}=\mathcal{R}$,

(iii) $(p \mathcal{R}+q \mathcal{R}) \cap(\bar{p} \mathcal{R}+\bar{q} \mathcal{R})=\mathcal{R},(p \mathcal{R}+\bar{q} \mathcal{R}) \cap(\bar{p} \mathcal{R}+q \mathcal{R})=\mathcal{R}$,

(iv) $p \mathcal{R}+q \mathcal{R}=\mathcal{R}, p \mathcal{R}+\bar{q} \mathcal{R}=\mathcal{R}, \bar{p} \mathcal{R}+q \mathcal{R}=\mathcal{R}, \bar{p} \mathcal{R}+\bar{q} \mathcal{R}=\mathcal{R}$.

Proof. (i) $\Rightarrow$ (ii): Assume $(p q-q p) \mathcal{R}=\mathcal{R}$. By Theorem 2.1 (iv) we have $b b^{\dagger}+b^{\dagger} b=1$, which implies $b b^{\dagger}=p$ and $b^{\dagger} b=\bar{p}$. Obviously $p-a \in p \mathcal{R}$, which shows $(p-a)(p-a)^{\dagger} \mathcal{R}=(p-a) \mathcal{R} \subseteq p \mathcal{R}$. By Lemma 2.2 (ii) we get $p=b b^{\dagger}=(p-a)(p-a)^{\dagger} b b^{\dagger}$, which proves $p \mathcal{R} \subseteq(p-a)(p-a)^{\dagger} \mathcal{R}$. Since $p \mathcal{R}=(p-a)(p-a)^{\dagger} \mathcal{R}$, then $p=(p-a)(p-a)^{\dagger}$. Also, from $b^{\dagger} b=\bar{p}$ and Lemma 2.2 (iii), we get $\bar{p} d d^{\dagger}=\bar{p}$, hence $d d^{\dagger}=\bar{p}$. From Theorem 2.1 (iii) we get $(p-q)(p-q)^{\dagger}=(p-a)(p-a)^{\dagger}+d d^{\dagger}=p+\bar{p}=1$, which yields $(p-q) \mathcal{R}=\mathcal{R}$. Furthermore, since $(p q-q p) \mathcal{R}=(p-q) \mathcal{R}$, by Theorem 4.6 we get $(1-p-q) \mathcal{R}=\mathcal{R}$.

(ii) $\Rightarrow$ (i) is evident in view of Theorem 4.6.

(ii) $\Leftrightarrow$ (iv): By Theorem 4.1 (iii), we have $(p-q)(p-q)^{\dagger}=(p-a)(p-$ $a)^{\dagger}+d d^{\dagger}$ and $(1-p-q)(1-p-q)^{\dagger}=(1-p-d)(1-p-d)^{\dagger}+a a^{\dagger}$. Hence (ii) is equivalent to the following

$$
(p-a)(p-a)^{\dagger}=p, d d^{\dagger}=1-p, a a^{\dagger}=p \text { and }(1-p-d)(1-p-d)^{\dagger}=1-p .
$$


From Lemma 4.2 , we can conclude that $p \mathcal{R}+q \mathcal{R}=\mathcal{R}$ if and only if $\bar{p}(\bar{p} q)^{\dagger}=\bar{p}$. By Theorem 4.1 (ii), we have that $\bar{p}(\bar{p} q)^{\dagger}=d d^{\dagger}$. Hence, $p \mathcal{R}+q \mathcal{R}=\mathcal{R}$ if and only if $d d^{\dagger}=1-p$. Similarly, changing $p, q$ with $1-p, 1-q$, respectively, we get the following

$$
\begin{array}{rll}
(1-p) \mathcal{R}+q \mathcal{R}=\mathcal{R} & \Leftrightarrow a a^{\dagger}=p, \\
p \mathcal{R}+(1-q) \mathcal{R}=\mathcal{R} & \Leftrightarrow \quad(1-p-d)(1-p-d)^{\dagger}=1-p, \\
(1-p) \mathcal{R}+(1-q) \mathcal{R}=\mathcal{R} & \Leftrightarrow \quad(p-a)(p-a)^{\dagger}=p .
\end{array}
$$

(iii) $\Leftrightarrow$ (iv) is evident.

Theorem 4.8. Let $\mathcal{R}$ be $a *$-reducing ring and $p, q \in \mathcal{R}$ be two projections such that $p q \bar{p}$ is Moore-Penrose invertible. Then

(i) $(p q-q p) \mathcal{R}=p q \bar{p} \mathcal{R} \oplus^{\perp} \bar{p} q p \mathcal{R}$,

(ii) $(p q-q p)^{\circ}=(p q \bar{p})^{\circ} \cap(\bar{p} q p)^{\circ}$.

Proof. (i): By Theorem 4.1 (iv), $(p q-q p) \mathcal{R}=(p q-q p)(p q-q p)^{\dagger} \mathcal{R}=$ $\left(b b^{\dagger}+b^{\dagger} b\right) \mathcal{R}$. Since, $b b^{\dagger} \in p \mathcal{R} p$ and $b^{\dagger} b \in \bar{p} \mathcal{R} \bar{p}$, we get that $\left(b b^{\dagger}\right)\left(b^{\dagger} b\right)=$ $\left(b^{\dagger} b\right)\left(b b^{\dagger}\right)=0$ which implies that $(p q-q p) \mathcal{R}=b b^{\dagger} \mathcal{R}+b^{\dagger} b \mathcal{R}=b \mathcal{R}+b^{*} \mathcal{R}$ and furthermore that this sum is orthogonal.

(ii): By Theorem 4.1 (iv) and by Lemma 2.1 we have

$$
\begin{aligned}
(p q-q p) x=0 & \Longleftrightarrow(p q-q p)^{\dagger}(p q-q p) x=0 \quad \Longleftrightarrow \quad\left(b b^{\dagger}+b^{\dagger} b\right) x=0 \\
& \Longleftrightarrow b b^{\dagger} x=0 \text { and } b^{\dagger} b x=0 \quad \Longleftrightarrow \quad b^{\dagger} x=0 \text { and } b x=0 \\
& \Longleftrightarrow b^{*} x=0 \text { and } b x=0 .
\end{aligned}
$$

Let us remark that when $a$ and $b$ are Hermitian commuting elements of a ring with involution, the reverse order law for the Moore-Penrose inverse holds, i.e. $(a b)^{\dagger}=b^{\dagger} a^{\dagger}$ (see e.g., [3, Th. 6.3.2]).

Theorem 4.9. Let $\mathcal{R}$ be $a *$-reducing ring and $p, q \in \mathcal{R}$ be two projections such that pqp, $p-p q p$ and $\bar{p} q \bar{p}$ are Moore-Penrose invertible. Then

(i) $p q \mathcal{R}+(p-q) \mathcal{R}=(p+q) \mathcal{R}$, 
(ii) $p q \mathcal{R} \cap(p-q) \mathcal{R}=\{0\}$ if and only if $p q$ is a projection.

Proof. (i): By Lemma 4.2, we have that

$$
x=p q(p q)^{\dagger}+\left(1-p q(p q)^{\dagger}\right)\left(\left(1-p q(p q)^{\dagger}\right)(p-q)(p-q)^{\dagger}\right)^{\dagger}
$$

is a projection and that $x \mathcal{R}=p q \mathcal{R}+(p-q) \mathcal{R}$. By Corollary 4.1, we have that $(p q)^{\dagger}=q(p q p)^{\dagger}$ which using the notations introduced in (2.3), implies that $p q(p q)^{\dagger}=p q p(p q p)^{\dagger}=a a^{\dagger}$. Also, by Theorem 4.1 (iii), we have that $(p-q)(p-q)^{\dagger}=(p-a)(p-a)^{\dagger}+d d^{\dagger}$. Now,

$$
\begin{aligned}
x & =a a^{\dagger}+\left(1-a a^{\dagger}\right)\left(\left(1-a a^{\dagger}\right)\left((p-a)(p-a)^{\dagger}+d d^{\dagger}\right)\right)^{\dagger} \\
& =a a^{\dagger}+\left(1-a a^{\dagger}\right)\left(\left(1-a a^{\dagger}\right)(p-a)(p-a)^{\dagger}+d d^{\dagger}\right)^{\dagger} \\
& =a a^{\dagger}+\left(1-a a^{\dagger}\right)\left(\left(p-a a^{\dagger}\right)(p-a)^{\dagger}+d d^{\dagger}\right)^{\dagger} .
\end{aligned}
$$

Since $\left(p-a a^{\dagger}\right)(p-a)^{\dagger} \in p \mathcal{R} p$ and $d d^{\dagger} \in \bar{p} \mathcal{R} \bar{p}$, we have that $\left(\left(p-a a^{\dagger}\right)(p-\right.$ $\left.a)^{\dagger}+d d^{\dagger}\right)^{\dagger}=\left(\left(p-a a^{\dagger}\right)(p-a)^{\dagger}\right)^{\dagger}+\left(d d^{\dagger}\right)^{\dagger}=\left(\left(p-a a^{\dagger}\right)(p-a)^{\dagger}\right)^{\dagger}+d d^{\dagger}$. Since $a a^{\dagger}=p a a^{\dagger}=a a^{\dagger} p$, it follows that $p-a a^{\dagger}$ is a projection, which implies that $\left(p-a a^{\dagger}\right)^{\dagger}=p-a a^{\dagger}$. Now, using the reverse order law for the Moore-Penrose inverse and the fact that $a, a^{\dagger}$ commute, we get that $\left(\left(p-a a^{\dagger}\right)(p-a)^{\dagger}\right)^{\dagger}=(p-a)\left(p-a a^{\dagger}\right)=p-a-a a^{\dagger}+a a a^{\dagger}=p-a a^{\dagger}$. Hence,

$$
x=a a^{\dagger}+\left(1-a a^{\dagger}\right)\left(p-a a^{\dagger}+d d^{\dagger}\right)=p+d d^{\dagger} .
$$

Now, by Theorem 4.1 (i), it follows that $x=(p+q)(p+q)^{\dagger}$, i.e. $x \mathcal{R}=(p+q) \mathcal{R}$.

(ii) From Lemma 4.2, we have that

$$
y=p q(p q)^{\dagger}-p q(p q)^{\dagger}\left(p q(p q)^{\dagger}\left(1-(p-q)(p-q)^{\dagger}\right)\right)^{\dagger}
$$

is a projection and that $y \mathcal{R}=p q \mathcal{R} \cap(p-q) \mathcal{R}$. As in the proof of item (i), we have that

$$
\begin{aligned}
y & =a a^{\dagger}-a a^{\dagger}\left(a a^{\dagger}\left(1-(p-a)(p-a)^{\dagger}-d d^{\dagger}\right)\right)^{\dagger} \\
& =a a^{\dagger}-a a^{\dagger}\left(a a^{\dagger}-a a^{\dagger}(p-a)(p-a)^{\dagger}\right)^{\dagger} .
\end{aligned}
$$


By Lemma 2.4 (i) and Lemma $2.3(\mathrm{i})$, we have that $a a^{\dagger}-a a^{\dagger}(p-a)(p-a)^{\dagger}=$ $p-(p-a)(p-a)^{\dagger}$ which is a projection, so we get that

$$
y=a a^{\dagger}-a a^{\dagger}\left(p-(p-a)(p-a)^{\dagger}\right)=a a^{\dagger}(p-a)(p-a)^{\dagger} .
$$

By (4.3), we have that $y=b b^{\dagger}$. Now, $p q \mathcal{R} \cap(p-q) \mathcal{R}=\{0\}$ if and only if $y=0$ if and only if $b=0$ which is equivalent to $p q=q p$. Finally, since $p, q$ are projections, $p q=q p$ is equivalent to the fact that $p q$ is a projection.

Theorem 4.10. Let $\mathcal{R}$ be a $*$-reducing ring and $p, q \in \mathcal{R}$ be two projections such that $p \bar{q} p$ and $\bar{p} q \bar{p}$ are Moore-Penrose invertible. Then the following conditions are equivalent:

(i) $p \mathcal{R} \cap q \mathcal{R}=\{0\}$,

(ii) $(p-q) \mathcal{R}=p \mathcal{R}+q \mathcal{R}$,

(iii) $p \bar{q} \mathcal{R} \oplus^{\perp} \bar{p} q \mathcal{R}=p \mathcal{R}+q \mathcal{R}$.

Proof. By Lemma 4.2 (ii), it follows that $p \mathcal{R} \cap q \mathcal{R}=\{0\}$ is equivalent to $p=p(p \bar{q})^{\dagger}$. Now, by using the representations of $p, q$ given in (2.2) we have

$$
p(p \bar{q})^{\dagger}=p(p \bar{q})^{*}\left[p \bar{q}(p \bar{q})^{*}\right]^{\dagger}=p \bar{q} p(p \bar{q} p)^{\dagger}=(p-a)(p-a)^{\dagger} .
$$

Hence, (i) is equivalent to $p=(p-a)(p-a)^{\dagger}$.

For the element $x$ defined in item (i) of Lemma 4.2, we have that

$$
x=p+\bar{p} q(\bar{p} q)^{\dagger}=p+\bar{p} q(\bar{p} q)^{*}\left[\bar{p} q(\bar{p} q)^{*}\right]^{\dagger}=p+\bar{p} q \bar{p}(\bar{p} q \bar{p})^{\dagger}=p+d d^{\dagger} .
$$

By (4.7) and Theorem 4.1 (iii) one has

$$
(p-q)(p-q)^{\dagger}=(p-a)(p-a)^{\dagger}+d d^{\dagger}=(p-a)(p-a)^{\dagger}+x-p .
$$

Thus, (i) is equivalent to $(p-q)(p-q)^{\dagger}=x$.

(i) $\Rightarrow$ (ii): If $(p-q)(p-q)^{\dagger}=x$, then by Lemma 4.2 (i), one has $(p-q) \mathcal{R}=(p-q)(p-q)^{\dagger} \mathcal{R}=x \mathcal{R}=p \mathcal{R}+q \mathcal{R}$.

(ii) $\Rightarrow$ (i): From the hypothesis and Lemma 4.2 (i), we get $(p-q)(p-$ $q)^{\dagger} \mathcal{R}=x \mathcal{R}$. Since both $(p-q)(p-q)^{\dagger}$ and $x$ are projections we get $(p-$ $q)(p-q)^{\dagger}=x$. 
(ii) $\Leftrightarrow$ (iii): This part follows by Theorem 3.1.

\section{Acknowledgments}

The authors would like to thank the anonymous reviewer for his $\backslash$ her useful suggestions, which helped to improve the original version of this paper.

\section{References}

[1] O.M. Baksalary, G. Trenkler, Column space equalities for orthogonal projectors, Appl. Math. Comput. 212 (2009) 519-529.

[2] J. Benítez, Moore.Penrose inverses and commuting elements of $\mathrm{C}^{*}$ algebras, J. Math. Anal. Appl., 345 (2008) 766-770.

[3] D.S. Djordjević and V. Rakočević, Lectures on Generalized Inverses, Faculty of Sciences and Mathematics, Niš.

[4] J.A. Green, On the structure of semigroups, Ann. of Math. (2) 54, (1951) 163-172.

[5] X. Mary, On generalized inverses and Green's relations, Linear Algebra Appl. 434(8) (2011) 1836-1844.

[6] R.E. Harte, M. Mbekhta, On generalized inverses in $C^{*}$-algebras, Studia Math. 103 (1992), 71-77.

[7] R.E. Harte and M. Mbekhta, On generalized inverses in $C^{*}$-algebras, II , Studia Math. 106 (1993) 129-138.

[8] J.J. Koliha, The Drazin and Moore-Penrose inverse in $C^{*}$-algebras, Proc. R. Ir. Acad. 99A (1999) 17-27.

[9] J.J. Koliha and V. Rakočević, Invertibility of the difference of idempotents, Linear and Multilinear Algebra 51 (2003) 97-110.

[10] J.J. Koliha, Elements of $C^{*}$-algebras commuting with their MoorePenrose inverse, Studia Math. 139 (2000) 81-90. 
[11] J.J. Koliha, Range projections of idempotents in $C^{*}$-algebras, Demonstr. Math. 34 (2001) 91-103.

[12] J.J. Koliha, D. Cvetković-Ilić, D. Chunyuan, Generalized Drazin invertibility of combinations of idempotents, Linear Algebra Appl. 437(9) (2012) 2317-2324.

[13] V.J. Neumann, On Regular Rings, Proc. Nat. Acad. Sci. USA 22 (1936) 707-712.

[14] R. Piziak, P.L. Odell, R. Hahn, Constructing projections on sums and intersections, Comput. Math. Appl. 37 (1999) 67-74.

[15] P. Patricio, R. Puystjens, Drazin-Moore-Penrose invertibility in rings, Linear Algebra Appl. 389 (2004) 159-173.

[16] V. Rakočević, Moore-Penrose inverse in Banach algebras, Proc. R. Ir. Acad. 88A (1988) 57-60. 